\title{
Fertility of HIV-infected women: insights from Demographic and Health Surveys
}

\author{
Wei-Ju Chen, Neff Walker
}

- An additional supplemental file published online only. To view this file please visit the journal online (http://sti.bmj.com).

Department of International Health, Johns Hopkins Bloomberg School of Public Health, Maryland, USA

\section{Correspondence to} Dr Neff Walker, Johns Hopkins Bloomberg School of Public Health, $615 \mathrm{~N}$ Wolfe Street, Baltimore, MD 21205, USA; pneffwalker@yahoo.com

Accepted 26 July 2010

\section{ABSTRACT}

Objective To analyse the data from Demographic and Health Survey (DHS) linking HIV serological data and fertility, and to recommend new age-specific fertility rate (ASFR) ratios of HIV-positive women to HIV-negative women to be used in the WHO/UNAIDS HIV estimates. Methods The authors obtained ASFRs and ASFR ratios from HIV-infected women and HIV-uninfected women from 20 DHS surveys collected between 2003 and 2007. The authors then computed mean and median ASFR ratios, and performed general linear modelling to estimate the overall ASFR ratio for each age group, and finally performed an analysis to identify determinants to national ASFR ratio for women aged 15-19 years old.

Results The overall ASFR ratio estimated by regression was highest among women aged 15-19 years old (ASFR ratio of 1.20), and decreased with age (ASFR ratio of $0.76,0.71,0.65,0.59$ and 0.53 , respectively for every 5 -year bracket of 20-44 years old). The mean and median of ASFR ratios among 20 countries were similar to the results above. The analysis suggested that country-specific proportion of sexually active women aged 15-19 years old could be a determinant of the ASFR ratio for this group. The higher proportion of sexually active women in this group suggested a lower ASFR ratio in young women.

Conclusion This study confirmed previous findings of a high ASFR ratio among women younger than 20 years old, and of decreasing ASFR ratios with age among those aged 20 years or older, based on nationally representative data from a large number of countries. The study updated the assumptions used in the annual WHO/UNAIDS HIV epidemic estimates. Countries should compute their own ASFR ratio of 15-19-year-old women accounting for the sexually active rate among young females.

\section{INTRODUCTION}

Surveillance of HIV infection in antenatal clinics (ANCs) has been the main system of monitoring the HIV epidemic since the mid-1980s in countries with generalised epidemics. This population was used for surveillance primarily because of easy access to women attending clinics, and unlike most other populations in health settings, there would not be a bias due to illness related to HIV status. Moreover, since most pregnant women have blood drawn for syphilis testing, remaining blood could be tested for HIV without informed consent. ${ }^{1}{ }^{2}$ Although population-based surveys do provide more representative estimates for monitoring HIV epidemics, the number of countries with these data sets is still small.
UNAIDS and WHO publish estimates on the global HIV epidemic that show the current status and trends of HIV worldwide based on the best available country data. In general, national estimates of adult prevalence in countries with generalised epidemics have been based on HIV prevalence among pregnant women attending ANCs, and these values, along with other data and assumptions are used to estimate other HIV and AIDS outputs such as incidence, mortality and need for treatment in both adults and children. ${ }^{3}$ One such adjustment is the relative age-specific fertility rates of women who are HIV-positive compared with those of their HIV-negative peers. This adjustment helps to account for the probability of women presenting to ANCs and is critical in making estimates of children born to HIV-positive women along with estimates of children needing treatment and dying of AIDS. ${ }^{4}$

Numerous studies have shown that HIV infection reduces fertility. ${ }^{1}{ }^{5-7}$ In previous UNAIDS estimates, the fertility ratio among HIV-positive women compared with women without HIV was assumed to be 0.7 for women older than 20 years. For women aged 15-19, the status of HIV infection was an indication that a woman was sexually active and thus had a higher probability of being pregnant than HIV-negative women of the same age. As a result, the fertility adjustment was set at 1.5 , that is, fertility was assumed to be $50 \%$ higher among HIV-positive compared with HIV-negative women. ${ }^{3} 8$

The value used for the previous assumptions was based on smaller community-based surveys. ${ }^{1}{ }^{5-8}$ However, in the last 7 years, 25 countries have conducted population-based surveys, such as Demographic and Health Surveys (DHS) or AIDS indicator surveys (AIS), which provide national representative information regarding HIV prevalence. Moreover, a recent study based on DHS in Cameroon in $2004^{9}$ showed that age-specific fertility rate is lower in HIV-positive women than HIV-negative women across 15 to 49-year-olds when restricted to the sexually active women population. The fertility rate ratio in this study was restricted to sexually active women aged 15 to 49 years old which is different from the whole women population used for adjusting fertility in UNAIDS estimates. Therefore, updated analyses based on population-based surveys are necessary.

Unlike older women, most of whom were sexually active and may have already acquired HIV infection in their earlier life stage, selection bias of women presenting to ANCs and acquiring HIV infection is more a concern for young women aged $15-19$ years old and may vary in different countries. 
Previous studies showed a higher ASFR ratio of HIV-positive to HIV-negative women aged 15-19 years old when the median age of first sexual intercourse is older, ${ }^{10} 11$ while a relative stable ASFR ratio was observed for women aged 20 years and older. The explanation for this is that when there is a later age of sexual debut, young women (15-19 years old) who are HIV-positive are a selected population in terms of sexual activity and would have a higher fertility than their peers.

In this paper, we computed ASFR and ASFR ratios for HIV-positive women and HIV-negative women for countries with national survey data. By combining the data from all countries, we derived an overall estimate of the ASFR ratios. The primary output of the analysis was ASFR ratios. Additionally, we investigated the impact of the level of sexual activity among the youngest age group on the fertility ratio between HIV-positive and HIV-negative women, and investigated whether the proportion of sexually active women in young group could be a good proximate determinant for ASFR ratio in countries with a diverse pattern of teenager sexual activity.

\section{METHODS}

\section{Data source}

Since 2001, more than 20 countries in Sub-Saharan Africa (SSA), the Caribbean and South-East Asia (SEA) have conducted national population-based surveys, including Demographic and Health Surveys (DHS) and AIDS indicator surveys (AIS) that included HIV serological testing. The procedures of sampling, data collection, HIV testing and laboratory diagnosis of DHS have been clearly described elsewhere. ${ }^{12}$ By October 2008, 27 DHS and AIS surveys from 25 countries have both basic household surveys and HIV testing results. Surveys in Dominican Republic (2002), Mali (2001) and Zambia (2001-2002) were excluded due to a lack of dataset identification variables linking demographic data to HIV testing results; surveys in Uganda (2004) and Vietnam (2005) were excluded, since the HIV testing data are not available in public domain; AIS in Cote d'Ivoire (2004) and Tanzania (2003) were excluded, since the AIS survey did not collect fertility information. In total, 20 DHS surveys from countries in SSA, Caribbean, and SEA were available for analysis. ${ }^{13}$ Table 1 shows the basic information including survey year, number of women interviewed, number of women receiving HIV testing, national HIV prevalence and proportion of sexually active women aged 15-19 years old for the 20 surveys included in this analysis.

\section{Definition of fertility}

Age-specific fertility rate (ASFR) was defined as the total number of births in the 36 months preceding the survey divided by the sum of woman-years at child-bearing age during the same period multiplied by 1000 for every 5 -year age bracket. ${ }^{14}$ Women who were aged 15 to 49 years old at the time of interview were included. Calculation of ASFR was based on the following formula:

$$
\begin{aligned}
& \text { ASFR }_{\text {HIV-infected,age group a }}= \\
& \frac{\text { birth to HIV-infected women in the past } 36 \text { months }_{\text {age group a }}{ }^{14}}{\text { sum of women-years of HIV-infected women }} \text { age group a }
\end{aligned}
$$

We computed ASFR for HIV-positive women and HIV-negative

\begin{tabular}{|c|c|c|c|c|c|}
\hline Area country & $\begin{array}{l}\text { Survey } \\
\text { year }\end{array}$ & $\begin{array}{l}\text { Demographic and } \\
\text { Health Surveys } \\
\text { (women population) }\end{array}$ & $\begin{array}{l}\text { HIV testing } \\
\text { women } \\
\text { population }\end{array}$ & $\begin{array}{l}\text { National HIV } \\
\text { prevalence }\end{array}$ & $\begin{array}{l}\text { Percentage of sexually } \\
\text { active among women } \\
\text { aged } 15-19\end{array}$ \\
\hline \multicolumn{6}{|l|}{ Countries from SSA } \\
\hline \multicolumn{6}{|l|}{ Southern SSA } \\
\hline Swaziland & 2006 & 4987 & 4424 & 25.9 & 41.4 \\
\hline Lesotho & 2004 & 7905 & 3031 & 23.5 & 42.9 \\
\hline \multicolumn{6}{|l|}{ Eastern SSA } \\
\hline Zimbabwe & 2005 & 8907 & 6947 & 18.1 & 32.1 \\
\hline Malawi & 2004 & 11698 & 2686 & 11.8 & 52.2 \\
\hline Kenya & 2003 & 8195 & 3151 & 6.7 & 42.2 \\
\hline Rwanda & 2005 & 11321 & 5656 & 3.0 & 12.1 \\
\hline Ethiopia & 2005 & 14070 & 5736 & 1.4 & 27.7 \\
\hline \multicolumn{6}{|l|}{ Middle SSA } \\
\hline Cameroon & 2004 & 10656 & 5227 & 5.5 & 54.7 \\
\hline Congo Democratic Republic & 2007 & 9995 & 4492 & 1.3 & 52.3 \\
\hline \multicolumn{6}{|l|}{ Western SSA } \\
\hline Ghana & 2003 & 5691 & 5097 & 2.2 & 39.0 \\
\hline Burkina Faso & 2003 & 12477 & 4086 & 1.8 & 48.4 \\
\hline Liberia & 2007 & 7092 & 6381 & 1.6 & 73.3 \\
\hline Guinea & 2005 & 7954 & 3772 & 1.5 & 59.9 \\
\hline Mali & 2006 & 14583 & 4528 & 1.5 & 55.4 \\
\hline Niger & 2006 & 9223 & 4406 & 0.7 & 61.1 \\
\hline Senegal & 2005 & 14602 & 4278 & 0.7 & 29.1 \\
\hline \multicolumn{6}{|l|}{ Countries from outside SSA } \\
\hline \multicolumn{6}{|l|}{ Caribbean } \\
\hline Haiti & 2005 & 10757 & 5230 & 2.2 & 40.6 \\
\hline Dominican Republic & 2007 & 27195 & 24564 & 0.8 & 38.5 \\
\hline \multicolumn{6}{|l|}{ South-East Asia } \\
\hline Cambodia & 2005 & 16823 & 8047 & 0.6 & 10.9 \\
\hline India & 2005 & 124385 & 53332 & 0.3 & 27.8 \\
\hline
\end{tabular}
women separately. The ASFR ratio was defined as the ratio of

Table 1 Overview of available Demographic and Health Surveys by October 2008 
ASFRs among HIV-positive women divided by ASFRs among HIV-negative women for each of the 5-year age groups. This analysis was performed for two groups: (1) all women and (2) sexually active women.

\section{Overall ASFR ratio}

The overall ASFR ratio was calculated in two ways. First, we obtained the mean and median of the ASFR ratios for each age group. Box-and-whisker plots were constructed to show the mean and median of ASFR ratios for each age group, and the distribution of country-specific ASFR ratios in each age group. The second method was to obtain estimates of ASFR ratio over age by performing a general linear model (GLM) with random effect at the country level to account for the within-country correlation of ASFR ratios.

\section{Trend of ASFR ratios over age}

With this analysis we also wanted to examine the current assumption that the fertility reduction due to HIV is the same for all age groups beyond 15-19. Loess smoothing was applied to show the trend of ASFR ratios over age. To examine the trend of ASFR ratios, we performed general linear models with random effect at the country level as described above.

\section{Association of sexual activity with the ASFR ratio among 15-19-year-old women}

We defined sexually active women as those who have experienced sexual debut. Those who never had sex debut were sexually inactive. The variable 'age at first intercourse' in the DHS survey was used for this purpose. In this part of the analysis, we focused on teenagers aged 15-19 years old. Most of the women aged 20 years and older had already experienced their first intercourse. We computed the Pearson correlation coefficient ( $r$ ) to estimate the correlation between the ASFR ratio for women aged 15-19 years old and the proportion of sexually active women in the same age group. In addition, we performed simple linear regression to explore the association between the ASFR ratio and the proportion of sexually active female among those aged 15-19. The analysis was computed for all countries and for countries from SSA, separately. A prediction equation was suggested to estimate country-specific ASFR ratio for young women.

\section{RESULTS}

\section{ASFR and ASFR ratios}

Twenty DHS surveys were analysed, including 16 for countries of Sub-Saharan Africa, two from the Caribbean and two from South-East Asia. HIV prevalence in the 20 countries varied from $0.3 \%$ to $25.9 \%$ (table 1 ). Country-specific ASFR, and ASFR ratios of HIV infected women to HIV-negative women in 20 countries are presented in appendix 1. In general, the ASFR ratios were the highest in women aged 15-19 years old, and were mostly lower than 1 in the age groups above 20 years old. Some exceptions were observed-for example, ASFR ratios for 15-19-year-old group were lower than 1 in Congo Democratic Republic, Guinea, Liberia, Malawi and Niger.

We conducted a subgroup analysis on sexually active women to understand the impact of HIV infection on fertility among women at risk of pregnancy. ASFRs increased among the HIV-negative and young population (15-19 years old), and most ASFR ratios were lower than 1 (appendix 1).

\section{Overall ASFR ratios and trend of ASFR ratios over age}

To investigate ASFR ratios across all countries, we performed overall estimates of the ASFRs by combining data from all of
Table 2 Mean and median age-specific fertility rate ratio among all countries or among countries in Sub-Saharan Africa, along with total number of birth and number of countries with at least one birth to HIV-infected women in corresponding age groups

\section{(A) Among all women}

\begin{tabular}{|c|c|c|c|c|c|c|}
\hline \multirow[b]{3}{*}{ Age } & \multirow{2}{*}{\multicolumn{2}{|c|}{$\begin{array}{l}\text { No of births to } \\
\text { HIV-positive women } \\
\text { and no of countries } \\
\text { with at least one birth }\end{array}$}} & \multicolumn{4}{|c|}{$\begin{array}{l}\text { Age-specific fertility rate ratio among } \\
\text { all women }\end{array}$} \\
\hline & & & \multicolumn{2}{|c|}{$\begin{array}{l}\text { Among all } \\
\text { countries }(n=20)\end{array}$} & \multicolumn{2}{|c|}{$\begin{array}{l}\text { Among countries } \\
\text { in Sub-Saharan } \\
\text { Africa }(n=16)\end{array}$} \\
\hline & $\begin{array}{l}\text { Total no } \\
\text { of births }\end{array}$ & $\begin{array}{l}\text { No of } \\
\text { countries* }\end{array}$ & Mean & Median & Mean & Median \\
\hline $15-19$ & 307 & 19 & 1.24 & 1.22 & 1.07 & 1.18 \\
\hline $20-24$ & 757 & 20 & 0.74 & 0.67 & 0.70 & 0.67 \\
\hline $25-29$ & 612 & 20 & 0.72 & 0.73 & 0.71 & 0.73 \\
\hline $30-34$ & 455 & 20 & 0.69 & 0.66 & 0.72 & 0.67 \\
\hline $35-39$ & 195 & 17 & 0.58 & 0.64 & 0.64 & 0.66 \\
\hline $40-44$ & 45 & 13 & 0.51 & 0.43 & 0.59 & 0.53 \\
\hline $45-49$ & 4 & $2 \dagger$ & 0.23 & 0.00 & 0.29 & 0.00 \\
\hline
\end{tabular}

(B) Among sexually active women

\begin{tabular}{|c|c|c|c|c|c|c|}
\hline \multirow[b]{3}{*}{ Age } & \multirow{2}{*}{\multicolumn{2}{|c|}{$\begin{array}{l}\text { No of births to } \\
\text { HIV-positive women } \\
\text { and no of countries } \\
\text { with at least one birth }\end{array}$}} & \multicolumn{4}{|c|}{$\begin{array}{l}\text { Age-specific fertility rate ratio, among } \\
\text { sexually active women }\end{array}$} \\
\hline & & & \multicolumn{2}{|c|}{$\begin{array}{l}\text { Among all } \\
\text { countries }(n=20)\end{array}$} & \multicolumn{2}{|c|}{$\begin{array}{l}\text { Among countries } \\
\text { in Sub-Saharan } \\
\text { Africa }(n=16)\end{array}$} \\
\hline & $\begin{array}{l}\text { Total no } \\
\text { of births }\end{array}$ & $\begin{array}{l}\text { No of } \\
\text { countries* }\end{array}$ & Mean & Median & Mean & Median \\
\hline $15-19$ & 307 & 19 & 0.90 & 0.76 & 0.74 & 0.72 \\
\hline $20-24$ & 757 & 20 & 0.69 & 0.61 & 0.65 & 0.60 \\
\hline $25-29$ & 612 & 20 & 0.70 & 0.72 & 0.70 & 0.72 \\
\hline $30-34$ & 455 & 20 & 0.68 & 0.65 & 0.71 & 0.65 \\
\hline $35-39$ & 195 & 17 & 0.58 & 0.64 & 0.63 & 0.66 \\
\hline $40-44$ & 45 & 13 & 0.51 & 0.43 & 0.59 & 0.53 \\
\hline $45-49$ & 4 & $2 \dagger$ & 0.23 & 0.00 & 0.29 & 0.00 \\
\hline
\end{tabular}

*Number of countries with at least one birth to women with HIV infection in the corresponding age group.

†Both of these countries are in Sub-Saharan Africa.

the countries in two ways: the mean and median ASFRs and ratios of the countries, and estimates produced by regression method.

First, we computed mean and median of ASFR ratios for all countries and countries in SSA, respectively (table 2). Among all women, the mean and median ratios for all countries were 1.24 and 1.22 separately in the 15-19-year-old group, while both mean ratios and median ratios were between 0.7 and 0.51 for the older age groups. The distribution of ASFR ratios at a young age was much more diverse than those of other age groups shown in the box-and-whisker plots (figure 1). When the analysis was restricted to countries in Sub-Saharan Africa, the mean and median value of the ASFR ratio decreases to around 1.1 in the 15-19-year-old group. For other age groups, the mean and median ratios remain close to the values obtained from all 20 countries. The ASFR ratios decreased with age in groups older than 20 years old. In addition, the Loess smoothing curve suggested a declining ASFR ratio with increasing age in women aged 20 to 44 years old (figure 2). In the subgroup analysis on sexually active women, the means and medians of ASFR ratios were lower than 1 for all age groups, and decreased with age (table 2B).

The generalised linear model (GLM) that accounted for the within-country correlation of ASFR ratios showed a negative coefficient of -0.059 of ASFR ratios over age, indicating that ASFR ratios decrease by 0.059 with each shift in age group $(p=0.013)$. Predicted ASFR ratios based on the model are 0.765 , 


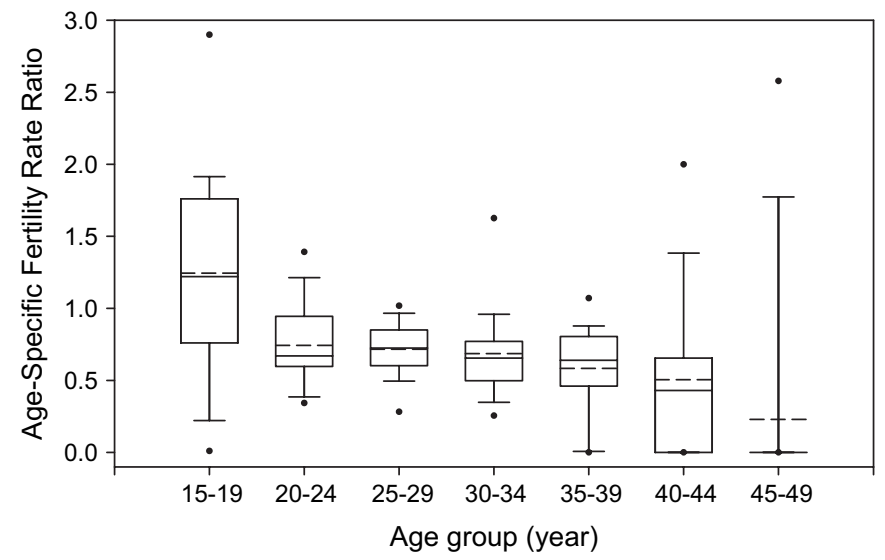

Figure 1 Box-and-whisker plot of age-specific fertility ratios among HIV-positive women to age-specific fertility rate among HIV-negative women. Solid line, median; dotted line, mean; dots, estimates outside the $95 \%$ percentile were presented.

$0.706,0.647,0.588,0.529$ and 0.47 for the six age groups beyond the age group 15-19 years old. The GLM estimates were similar to the Loess estimates (figure 2).

\section{Factor affecting ASFR ratio in young women aged $15-19$ years old}

Since the country ASFR ratios were heterogeneous among women aged 15-19 years old (figure 1), it was not appropriate to estimate an overall ASFR ratio in this age group. Instead, we investigated the correlation between level of sexual activity and ASFR ratio among young women. Among all 20 countries, the ASFR ratio was moderately to highly negatively correlated with the proportion of sexually active women aged 15-19 years old (figure $3 \mathrm{~A} ; \mathrm{r}=-0.6991,95 \% \mathrm{CI}-0.88$ to -0.38 ), while the negative correlation was stronger when restricted to countries in Sub-Saharan Africa (figure 3B; $r=-0.7923,95 \%$ CI -0.93 to $-0.49)$. The regression coefficient from simple linear regression was -0.031 for all countries ( $p=0.001,95 \%$ CI -0.046 to -0.015 ) and SSA countries ( $p=0.001,95 \% \mathrm{CI}-0.044$ to -0.017 ), indicating that the ASFR ratio would decrease by 0.31 with every $10 \%$ increase in proportion of sexually active women. The simple linear regression fitted for all countries was: ASFR

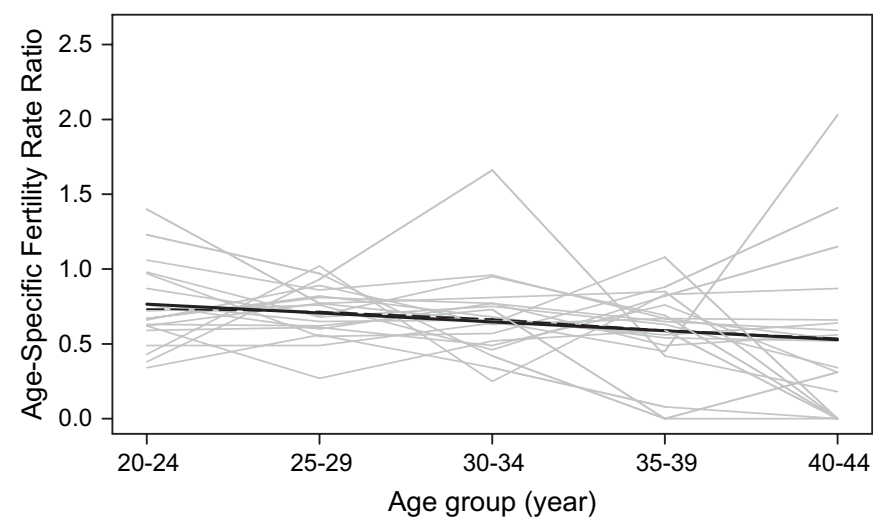

Figure 2 Age-specific fertility rate ratio of HIV-positive women to HIVnegative women and estimates of overall ratio produced by Loess smooth average and generalised linear models. Grey lines, countryspecific data; dashed line, Loess smoothing curve; solid line, GLM for age group 20-24 to 40-44 years old (ASFR

ratio $=0.8901-0.059^{*}$ group\#). Group\#: $2,20-24 ; 3,25-29$;

$4,30-34 ; 5,35-39 ; 6,40-44$.
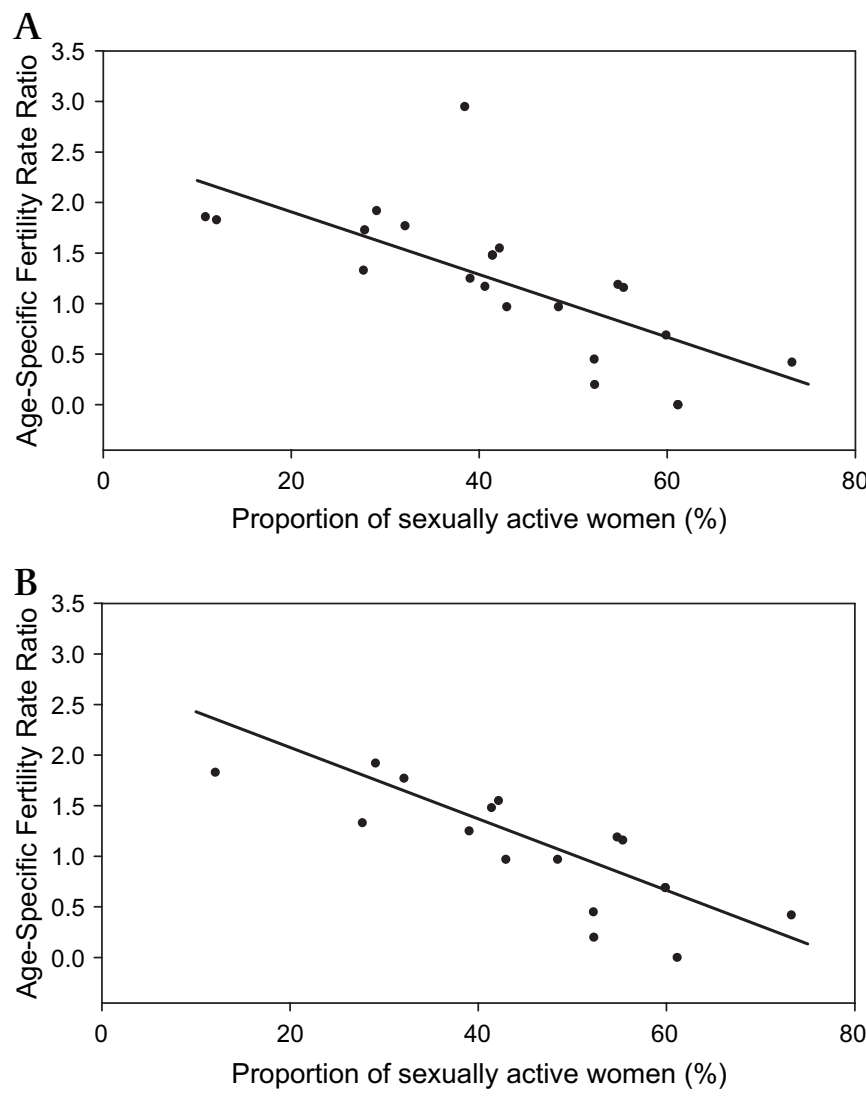

Figure 3 Age-specific fertility rate ratio and proportion of sexually active women in group aged $15-19$ years old. (A) All countries. (B) Countries in Sub-Saharan Africa.

ratio $=2.528-0.031 \times$ percentage of sexually active women aged 15-19 years old.

\section{DISCUSSION}

The study confirmed the UNAIDS assumptions of higher fertility for women 15-19 years old and lower fertility for women older than 20 years old for HIV-positive women in 20 national population-based surveys. The result also indicated that sexually active women with HIV infection had a lower fertility across all age groups compared with women who were sexually active but who were not infected with HIV. However, the analyses suggested that the previous assumptions, which set the ASFR ratio to 1.5 for $15-19$ years and 0.7 for the remaining age groups, ${ }^{3} 15$ could be improved. We recommended an updated fertility adjustment, $1.2,0.76,0.71,0.65,0.59,0.53$ and 0.47 , for each 5-year age bracket from 15-19, 20-24, 25-29, 30-34, 35-39, 40-44 and 45-49 years old, respectively. In addition, we proposed that the ASFR ratio in the 15-19-year-old age group should be adjusted based on the proportion of sexually active women in that age group if country-specific information is available.

One major concern of the study was the uncertain temporality of fertility events and HIV testing. Measurement of ASFR in DHS surveys was based on a retrospective report of fertility history in the past 36 months prior to the interview, while the HIV sero-positivity was confirmed at the time of survey by serological tests. We could not be sure when their sero-conversion occurred and could not identify the women's HIV status at the time of each conception. Therefore, some fertility events and women-years among HIV-negative women would be misclassified 
as HIV-positive, resulting in an underestimate of the impact of HIV infection on fertility by making the ASFRs in the two groups spuriously more similar. The longer the recall period, the greater the underestimation would be. On the other hand, using a shorter period of fertility recall, for example, 12 months prior to the interview, would greatly reduce the number of births, especially to HIV-positive women, across all age groups and reduce the precision of estimates. Therefore, we decided to use the standard ASFR definition in DHS surveys.

The study was the first one to utilise multiple large national surveys with HIV testing results to explore the association between HIV infection and fertility. Estimates were from national surveys which included larger populations, and were more reliable than the estimates from community-based studies. Systematic sampling and inclusion of different subpopulations produced representative estimates for the country. Careful design and training of those conducting the DHS surveys yielded data with good quality. ${ }^{12} 16$ Standardised questionnaires and interview procedures made the results from different countries comparable. All these merits add to the value of this analysis.

A previous study analysed 19 community-based studies using pregnancy data, mostly from a cross-sectional design, and suggested a series of ASFR ratios of HIV-positive to HIV-negative women (1.26 for 15-19 age group, 0.76 for 20-24 and 0.67 for 25-44 age groups). ${ }^{8}$ The estimates were similar to our estimates for the 15-29 age group, and the previous estimates were higher than ours for women aged $30-44$ years. We found a decreasing trend of ASFR ratios among adult women, while the previous study did not attempt to estimate the trend of ASFR ratios over age. Our analysis utilised DHS surveys which had the advantages of national surveys described in the last paragraph. In addition, this study included nationally representative surveys with large sample sizes and used cumulative incidence of fertility which yielded more accurate and precise estimates of women's fertility rate and rate ratio.

A lower fertility among HIV-infected women aged 20 years and older has been documented in many studies. ${ }^{15-7}$ Mechanisms involved in lowered fertility among HIV-positive women include reduced frequency of conception, increased fetal loss ${ }^{6} 1718$ and change in sexual behaviours-for example fewer sexual activities and more contraceptive use, ${ }^{19-21}$ and shortened life span of their HIV-positive partners. ${ }^{22} 23$ In addition, we suggested a decreasing ASFR ratio for women aged $20-44$ years across all countries. Age is one of the possible determinants associated with lower fertility. According to Ross et al, ${ }^{17} \mathrm{HIV}$-infected women with advanced disease had a greater fecundity loss. Since older HIV-infected women were more likely to have an advanced disease, the decreasing ASFR ratio with age could possibly result from an increased proportion of women with severe disease among the older groups.

Unlike the direct impact of HIV infection on reducing fertility among adult women, differential fertility among young women was associated with selection pressure of both pregnancy and HIV infection. The analyses also found a high correlation between ASFR ratio in women aged 15-19 in a country and the proportion of women aged 15-19 years old who were sexually active. Previous studies had suggested that this effect was primarily due to earlier sex debut, ${ }^{811}$ and so fertility rates among HIV-positive women in the 15-19-year age group may be higher than those of their HIV-negative peers due to differences in sexually activity. ${ }^{11124}$ The proportion of sexually active women aged 15-19 years old was used as an approximation of average age of sex debut in the population. We recommended that countries calculate their own ASFR ratio for the 15-19 age

\section{Key messages}

We found a decreasing trend of ASFR ratio over age among HIV-positive women to HIV-negative women.

- We recommend an updated set of assumptions relating to fertility and HIV status. We suggested the following ASFR ratio for each 5 -year-bracket group from $15-49$ years old: $1.2,0.765,0.706,0.647,0.588,0.529$ and 0.47 .

- We recommend that countries should compute their own ASFR ratio for 15-19 years old group based on the percent of sexually active women using the equation:

ASFR ratio $=2.528-0.031$

$\times$ percentage of sexually active women $_{15-19}$ years old

group based on sexual activity rate of women in this age group. For countries which do not have their own sexual active proportions, we recommended a general assumption of ASFR ratio as 1.2 for this age group

In conclusion, these analyses confirmed the previous assumption that HIV infected women aged 20 years and older have lower fertility compared with HIV-negative women. However, we suggested an updated series of ASFR ratios be used for the fertility adjustments in UNAIDS estimates. In the future the impact of treatment on the fertility of women with HIV should be investigated. As the provision of antiretroviral therapy (ART) becomes more widely available in low- and middleincome countries the assumptions about ASFR may need to be refined. Specifically ART could reduce illness of HIV-infected women and increase the fertility among older HIV-infected women. $^{25} 26$ Future work should address this issue as data become available.

Acknowledgements The authors thank R Ren from Macro International, who provided assistance and the SAS program package calculating age-specific fertility rate.

Funding This work was supported by UNAIDS.

Competing interests None.

Contributors W-JC contributed to the study design, carried out the data analyses and prepared the preliminary manuscript. NW contributed to the study design, supervised data analyses and edited the manuscript.

Provenance and peer review Commissioned; externally peer reviewed.

\section{REFERENCES}

1. Zaba B, Gregson S. Measuring the impact of HIV on fertility in Africa. Aids 1998; 12(Suppl 1):S41-50.

2. Chin J. Public health surveillance of AIDS and HIV infections. Bull World Health Organ 1990;68:529-36.

3. Stover J. Projecting the demographic consequences of adult HIV prevalence trends: the Spectrum Projection Package. Sex Transm Infect 2004;80(Suppl 1): i14-18.

4. Boerma JT, Stanecki KA, Newell ML, et al. Monitoring the scale-up of antiretroviral therapy programmes: methods to estimate coverage. Bull World Health Organ 2006;84:145-50.

5. Carpenter LM, Nakiyingi JS, Ruberantwari A, et al. Estimates of the impact of HIV infection on fertility in a rural Ugandan population cohort. Health Transit Rev 1997; 7(Suppl 2):113-26.

6. Gray RH, Wawer MJ, Serwadda D, et al. Population-based study of fertility in women with HIV-1 infection in Uganda. Lancet 1998;351:98-103.

7. Glynn JR, Buve A, Carael M, et al. Decreased fertility among HIV-1-infected women attending antenatal clinics in three African cities. J Acquir Immune Defic Syndr 2000;25:345-52.

8. Lewis JJ, Ronsmans C, Ezeh A, et al. The population impact of HIV on fertility in sub-Saharan Africa. Aids 2004;18(Suppl 2):S35-43

9. Kongnyuy EJ, Wiysonge CS. Association between fertility and HIV status: what implications for HIV estimates? BMC Public Health 2008;8:309. 
10. Zaba B, Pisani E, Slaymaker E, et al. Age at first sex: understanding recent trends in African demographic surveys. Sex Transm Infect 2004;80(Suppl 2):iï28-35.

11. Terceira N, Gregson S, Zaba B, et al. The contribution of HIV to fertility decline in rural Zimbabwe, 1985-2000. Popul Stud (Camb) 2003:57:149-64.

12. Mishra V, Vaessen M, Boerma JT, et al. HIV testing in national population-based surveys: experience from the Demographic and Health Surveys. Bull World Health Organ 2006;84:537-45.

13. ORC macro. Demographic and Health Survey and AIDS Indicator Survey. Calverton, MD: Macro International Inc, 2008.

14. ORC macro. Current Fertility Rates: Age Specific Fertility Rate. Calverton, MD: Macro International Inc (accessed 15 March 2010)

15. Morgan M, Walker N, Gouws E, et al. Improved plausibility bounds about the 2005 HIV and AIDS estimates. Sex Transm Infect 2006;82(Suppl 3):iii71-77.

16. Mishra V, Barrere B, Hong R, et al. Evaluation of bias in HIV seroprevalence estimates from national household surveys. Sex Transm Infect 2008;84(Suppl 1):i63-i70.

17. Ross A, Van der Paal L, Lubega R, et al. HIV-1 disease progression and fertility: the incidence of recognized pregnancy and pregnancy outcome in Uganda. Aids 2004:18:799-804.

18. Sedgh G, Larsen U, Spiegelman D, et al. HIV-1 disease progression and fertility in Dar es Salaam, Tanzania. J Acquir Immune Defic Syndr 2005:39:439-45.
19. Crampin AC, Jahn A, Kondowe M, et al. Use of antenatal clinic surveillance to assess the effect of sexual behavior on HIV prevalence in young women in Karonga district, Malawi. J Acquir Immune Defic Syndr 2008;48:196-202.

20. Urassa M, Kumogola $Y$, Isingo R, et al. HIV prevalence and sexual behaviour changes measured in an antenatal clinic setting in northern Tanzania. Sex Transm Infect 2006:82:301-6

21. Wilcher R, Cates W Jr, Gregson S. Family planning and HIV: strange bedfellows no longer. Aids 2009;23(Suppl 1):S1-6.

22. Gregson S, Zhuwau T, Anderson RM, et al. HIV and fertility change in rural Zimbabwe. Health Transit Rev 1997;7(Suppl 2):89-112.

23. Hunter SC, Isingo R, Boerma JT, et al. The association between HIV and fertility in a cohort study in rural Tanzania. J Biosoc Sci 2003;35:189-99.

24. Camlin CS, Garenne M, Moultrie TA. Fertility trend and pattern in a rural area of South Africa in the context of HIV/AIDS. Afr J Reprod Health 2004;8:38-54.

25. Bussmann H, Wester CW, Wester CN, et al. Pregnancy rates and birth outcomes among women on efavirenz-containing highly active antiretroviral therapy in Botswana. J Acquir Immune Defic Syndr 2007:45:269-73.

26. Myer L, Carter RJ, Katyal M, et al. Impact of antiretroviral therapy on incidence of pregnancy among HIV-infected women in Sub-Saharan Africa: a cohort study. PLoS Med 2010;7:e1000229. 